\title{
SALF et Recherche
}

\section{Etat des Lieux et Orientations Futures.}

\author{
Résultats d'un Questionnaire \\ adressé aux Membres de la Société
}

\begin{abstract}
Groupe de Travail sur la Recherche du Conseil d'Administration de la SALF*
*Jacques Auger, Serge Carreau, André Demoulin, Clément Jimenez et Jean Marc Rigot; depuis le CA du 29/01/2000 : Jacques Auger, Mohamed Benahmed, Serge Carreau, André Demoulin, Jean Marc Rigot et Patrick Thonneau
\end{abstract}

Au cours de la dernière décennie, la reconnaissance et le rayonnement de notre Société n'ont fait que croître, grâce notamment à la réussite de nos congrès, aux actions variées de formation continue ou encore, au support précieux de notre revue. Cependant, et particulièrement dans les pays de langue française, le domaine de l'Andrologie, pluridisciplinaire par nature, présente encore un impact réduit dans les priorités d'actions institutionnelles de recherche et de santé comparé à celui d'autres grands domaines de la biologie et de la médecine. Ceci pourrait, au moins en partie, expliquer le relativement faible nombre de travaux publiés portant aussi bien sur la physiologie, la physiopathologie, que les mécanismes moléculaires afférents qui émanent de groupes francophones en comparaison avec ceux provenant d'autres pays notamment de culture anglosaxonne.

Il est évident que notre Société doit apporter sa contribution au développement de la recherche et au soutien d'actions de recherche ayant trait à l'Andrologie, comme elle l'a fait par le passé pour l'enseignement de troisième cycle ou de formation continue.
Au cours de l'année 1999, le Conseil d'Administration de la SALF a retenu à l'unanimité, l'intérêt d'actions pour la recherche comme l'un des axes prioritaires parmi les orientations stratégiques de notre Société pour les années à venir. Un groupe de travail sur ce sujet a immédiatement été constitué.

Tous les membres de la SALF, dans la diversité de leur domaine (fertilité, sexualité,...), de leur pratique (clinique, recherche,..., public, privé,...), de leur spécialité (clinicien, biologiste, psy,...), sont potentiellement concernés par la recherche, l'implication personnelle dans une recherche pouvant aller d'une simple collaboration dans un protocole (par la réalisation et la transmission des résultats d'actes ou d'examens de routine prévus dans celui-ci) à un investissement complet dans l'élaboration, la réalisation et l'exploitation des résultats, qu'il s'agisse d'étude clinique ou expérimentale.

Pour que les futures actions en ce sens (par exemple, des études multicentriques, des soutiens spécifiques,....) ne soient pas seulement le reflet des préoccupations des membres du Conseil d'Administration, celui-ci a souhaité 
mieux connaître l'activité et les souhaits en matière de recherche de l'ensemble des membres de la SALF. Pour cela, un questionnaire bref leur a été adressé. Un résumé des réponses a été donné lors de l'Assemblée Générale des membres de la SALF à l'occasion du Congrès d'Issy les Moulineaux en décembre 1999.

Les questions portaient sur les statuts professionnels, les domaines de compétence, l'enseignement, le savoir faire (techniques de laboratoire, chirurgicales ou paracliniques maîtrisées), les axes de recherche en cours, les partenariats (transfert et valorisation, recherche clinique, etc...) et les projets et souhaits en matière de recherche.

Il y a eu 119 questionnaires reçus au 10/11/99, date limite d'envoi des réponses, ce qui représentait un taux de réponse de $32 \%$. Ce taux était à la fois intéressant, un tiers des membres de la SALF serait intéressé par des développements de recherche, et en même temps, malheureusement trop faible pour donner une véritable "photographie" de notre Société.

Les questions sur le statut, les domaines d'activité, les activités d'enseignement et les techniques maitrisées témoignaient de l'extraordinaire variété des "profils" des membres de la SALF (Figures 1, 2, 3, 4 et 5). Toutefois, on notera bien que les renseignements portés dans toutes les figures présentées ne concernent que les membres de la Société ayant répondu au questionnaire, d'une part, et que, d'autre part, seuls les totaux de chaque item répertorié sont présentés, les réponses très souvent multiples ne pouvant être illustrées de manière simple.

Concernant les recherches en cours, on a pu dénombrer 177 "thèmes" différents se basant sur les mots-clés tirés de ces réponses libres! Parmi ceux-ci, 61 étaient très vagues ou très généraux (exemple: recherche sur la spermatogénèse, le spermogramme ou la cytogénétique). Malgré cela on pouvait conclure que :

1) la très vaste majorité des membres ayant répondu étaient impliquées dans une recherche au moins,

2) beaucoup de membres ayant répondu étaient impliqués dans plus d'une recherche,
3) les thématiques annoncées étaient extrêmement variées et

4) il n'y avait pas de thématique se dégageant majoritairement quoique les réponses les plus fréquentes se rapportaient :

- au contrôle endocrinien de la spermatogénèse,

- aux déterminants génétiques et géniques de la spermatogénèse,

- au rôle de l'environnement sur la fonction de reproduction mâle,

- aux modulations du pouvoir fécondant des spermatozoïdes par son microenvironnement dans les voies génitales.

Pour les projets et souhaits en matière de recherche, cet item a été rempli par 64 des 119 membres ayant répondu (54\% des répondants, $17 \%$ par rapport au nombre total de membres). Le sens de la lettre d'accompagnement du questionnaire n'a peut être pas été tout à fait bien perçu : ce sont plus des thèmes de projet que des voeux de stratégie ou de support de la SALF qui ont été exprimés. Seulement 6 réponses faisaient référence à l'intérêt de bourses ou de financements pour des personnels techniques et dans 7 réponses, il y avait la notion de collaboration, enquête nationale ou encore étude multicentrique.

Là encore, les thématiques des projets semblaient en moyenne très intéressantes et variées pour les membres du groupe de travail mais il n'apparaissait pas comment la SALF pourrait apporter son soutien.

En conclusion, cette enquête n'a pas permis de donner une photographie réaliste de la SALF à cause du nombre insuffisant de réponses, mais ce n'était pas son objectif principal. Par contre, elle montrait bien que de très nombreux membres sont directement impliqués dans des actions de recherche très variées. Sur la base de l'échantillon ayant répondu, elle témoignait de l'extraordinaire faisceau de compétences multiples de ses membres. Ces résultats incitent à préciser la réflexion actuelle sur l'avenir de la recherche dans notre domaine et au sein de la Société d'Andrologie de Langue Française afin que cette dernière puisse proposer à ses membres des actions concrètes dans un futur proche. 


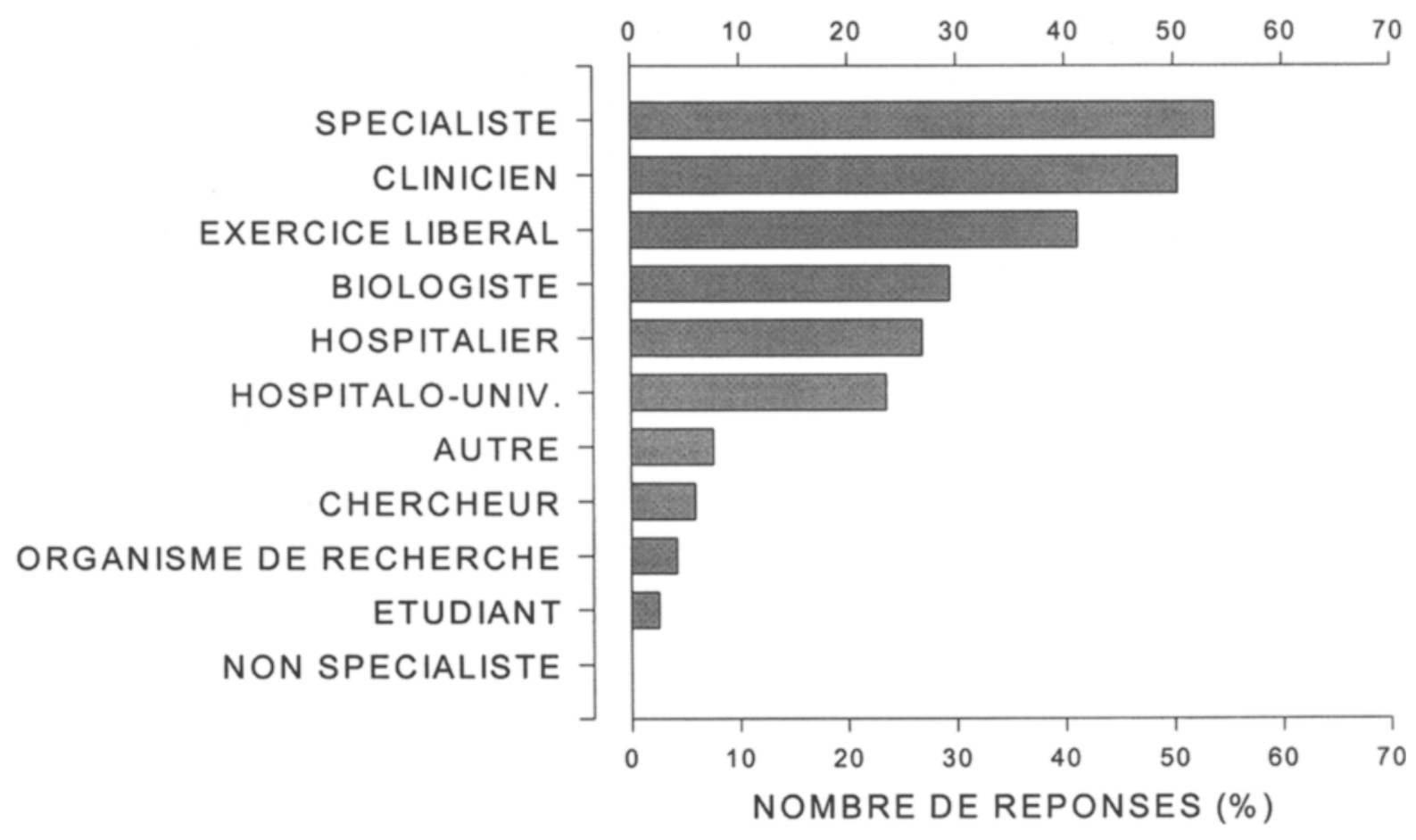

Figure 1 : Statuts professionnels

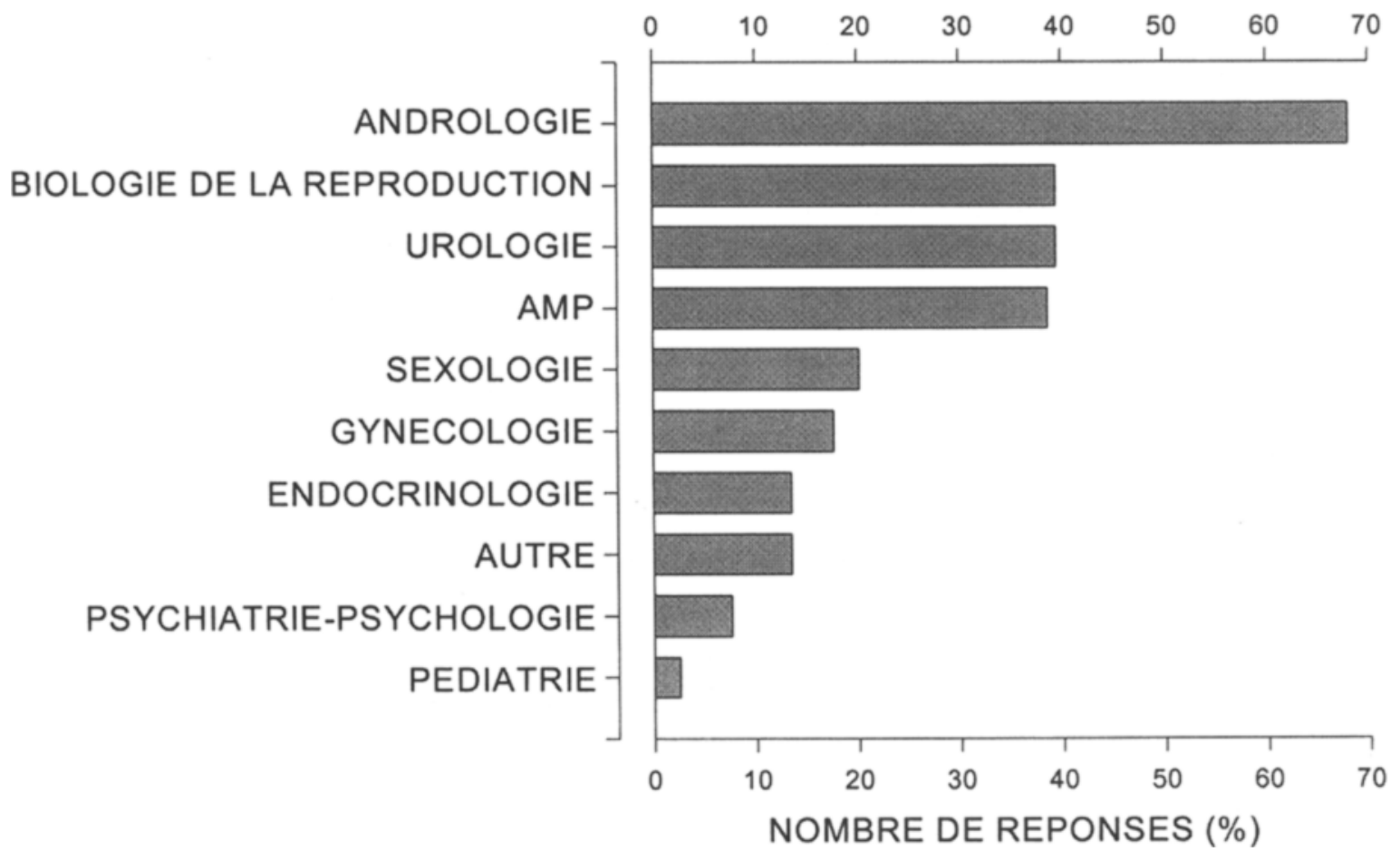

Figure 2: Domaines de compétences 

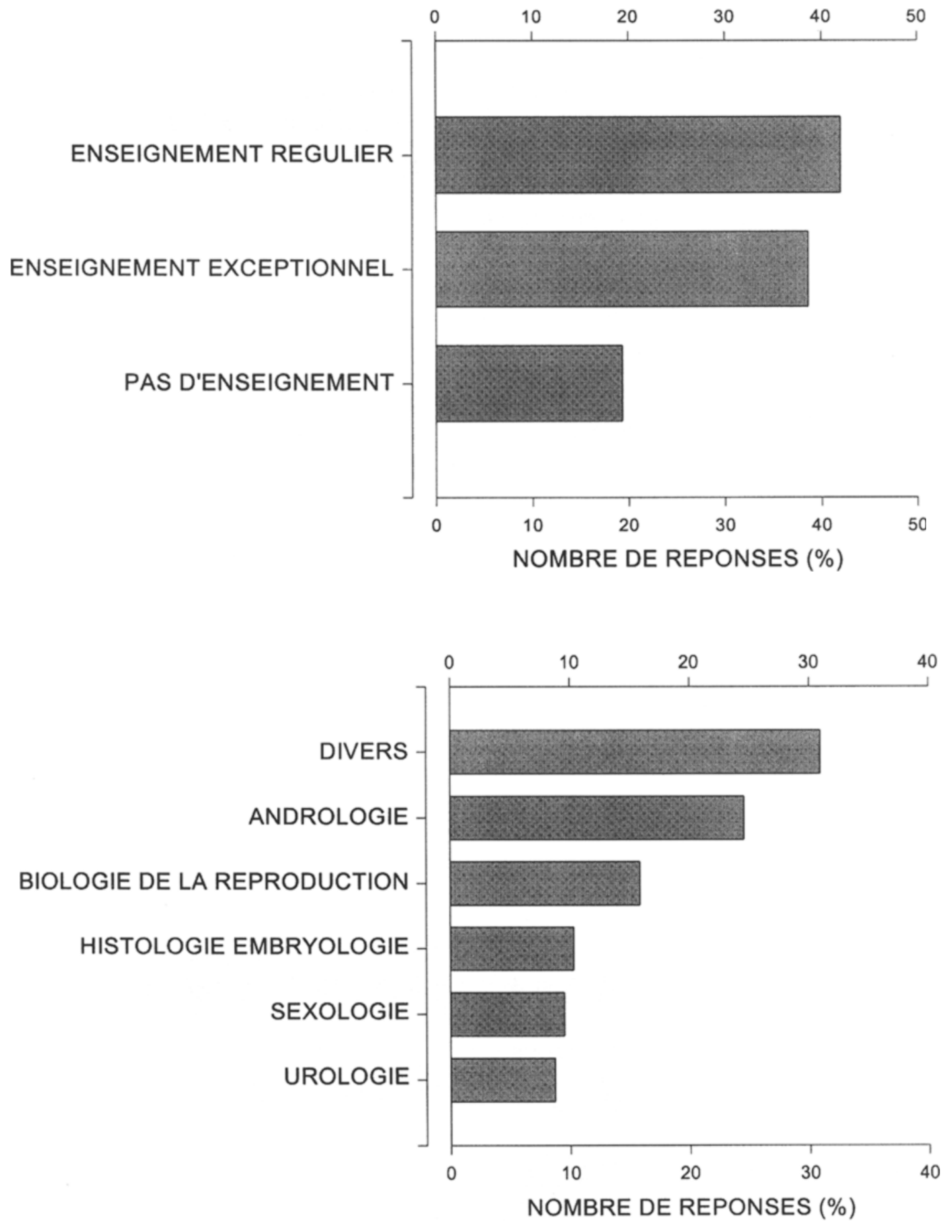

Figure 3: Activités d'enseignement 


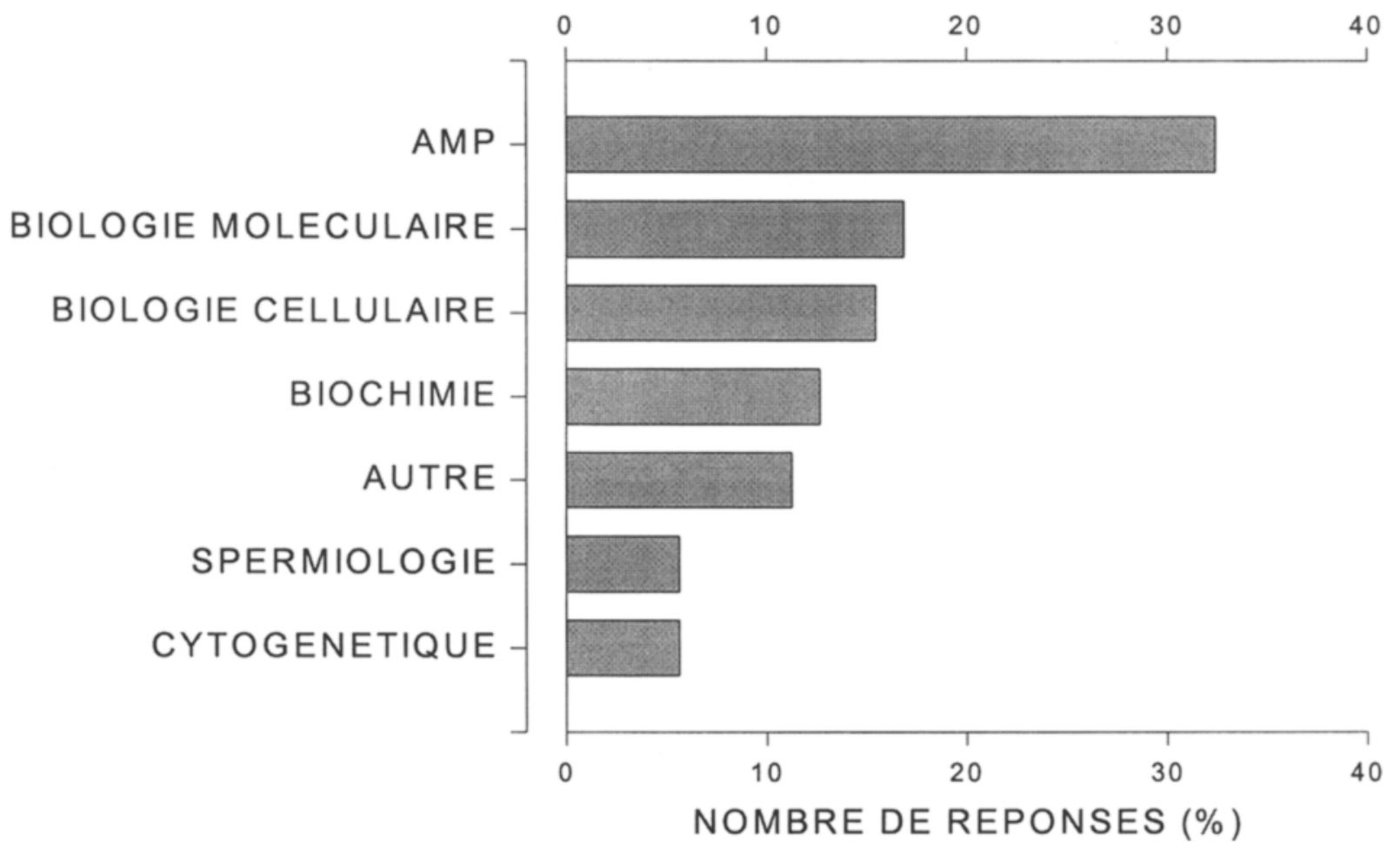

Figure 4. Techniques de laboratoire mâ̂trisées

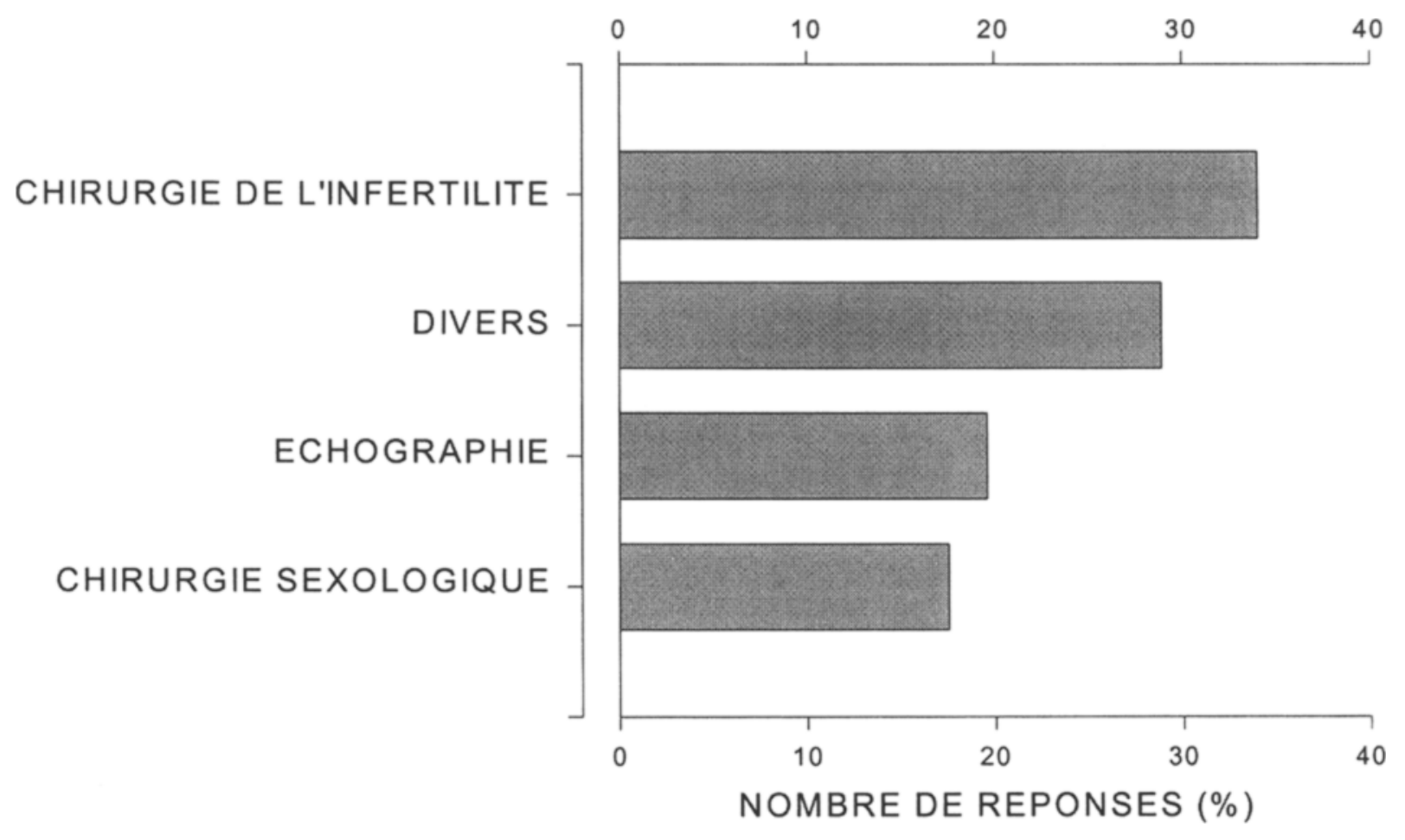

Figure 5. Techniques cliniques maîtrisées 